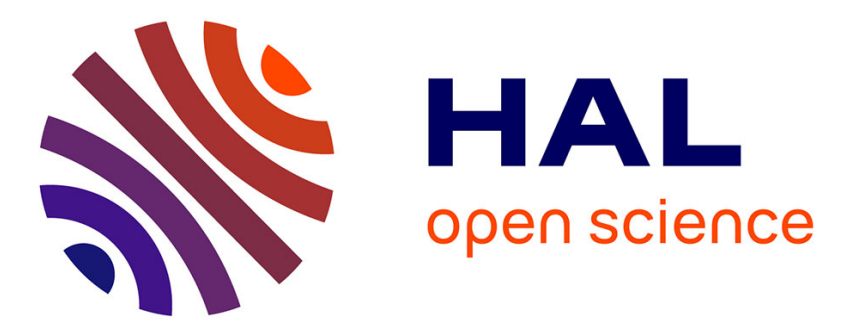

\title{
Intestinal dysbiosis in Inflammatory Bowel Disease associated with primary immunodeficiency.
}

Harry Sokol, Nizar Mahlaoui, Claire Aguilar, Perrine Bach, Olivier

Join-Lambert, Aurélie Garraffo, Philippe Seksik, François Danion, Sarah

Jegou, Marjolene Straube, et al.

\section{To cite this version:}

Harry Sokol, Nizar Mahlaoui, Claire Aguilar, Perrine Bach, Olivier Join-Lambert, et al.. Intestinal dysbiosis in Inflammatory Bowel Disease associated with primary immunodeficiency.. Journal of Allergy and Clinical Immunology, 2019, 143 (2), pp.1-10. 10.1016/j.jaci.2018.09.021 . hal-01913759

\section{HAL Id: hal-01913759 \\ https://hal.science/hal-01913759}

Submitted on 6 Nov 2018

HAL is a multi-disciplinary open access archive for the deposit and dissemination of scientific research documents, whether they are published or not. The documents may come from teaching and research institutions in France or abroad, or from public or private research centers.
L'archive ouverte pluridisciplinaire HAL, est destinée au dépôt et à la diffusion de documents scientifiques de niveau recherche, publiés ou non, émanant des établissements d'enseignement et de recherche français ou étrangers, des laboratoires publics ou privés. 
1 Intestinal dysbiosis in Inflammatory Bowel Disease associated with primary

2

3

4

5 immunodeficiency.

Harry Sokol, MD, $\mathrm{PhD}^{1,2,3,4}$, Nizar Mahlaoui, MD ${ }^{4,5,17}$, Claire Aguilar, $\mathrm{MD}, \mathrm{PhD}^{6,8,17}$, Perrine Bach, $\mathrm{MSc}^{4}$, Olivier Join-Lambert, MD, $\mathrm{PhD}^{7}$, Aurélie Garraffo,6, Philippe Seksik, MD, PhD 1,3, François Danion, MD, ${ }^{6}$, Sarah Jegou, MSc ${ }^{1}$, Marjolene Straube, MSc ${ }^{2}$, Christelle Lenoir, $\mathrm{MSc}^{8}$, Bénédicte Neven, MD, $\mathrm{PhD}^{5,8,9}$, Despina Moshous ${ }^{5,8,9} \mathrm{MD}, \mathrm{PhD}$, Stéphane Blanche, MD, $\mathrm{PhD}^{5,9}$, Bénédicte Pigneur, $\mathrm{MD}, \mathrm{PhD}^{8,10}$, Olivier Goulet, $\mathrm{MD}, \mathrm{PhD}^{8,10}$, Frank Ruemmele, MD, $\mathrm{PhD}^{8,10}$, Felipe Suarez, MD, $\mathrm{PhD}^{4,8,12}$, Laurent Beaugerie, $\mathrm{MD}, \mathrm{PhD}^{3}$, Stéphanie Pannier, $\mathrm{MD}, \mathrm{PhD}^{11,13}$, Françoise Mazingue, $\mathrm{MD}, \mathrm{PhD}^{14}$, Olivier Lortholary, $\mathrm{MD}, \mathrm{PhD}^{4,6,9}$, Lionel Galicier, $\mathrm{MD}, \mathrm{PhD}^{15}$, Capucine Picard, $\mathrm{MD}, \mathrm{PhD}^{8,9,11,4}$, Geneviève de Saint Basile, $\mathrm{MD}, \mathrm{PhD}$ 8,9,11, Sylvain Latour, $\mathrm{PhD}^{8,9}$, Alain Fischer, $\mathrm{MD}, \mathrm{PhD}^{4,5,8,9,16}$

${ }^{1}$ Sorbonne Universités, UPMC Univ. Paris 06, École normale supérieure, PSL Research University, CNRS, INSERM, APHP, Laboratoire des Biomolécules (LBM), 27 rue de Chaligny, 75005 Paris, France

${ }^{2}$ INRA, UMR1319 Micalis \& AgroParisTech, Jouy en Josas, France

${ }^{3}$ Department of Gastroenterology, Saint Antoine Hospital, AP-HP, UPMC Univ Paris 06, Paris, France

${ }^{4}$ French National Reference Center for Primary Immune Deficiency (CEREDIH), Necker Enfants Malades University Hospital, Assistance Publique-Hôpitaux de Paris (APHP), Paris, France

${ }^{5}$ Paediatric Haematology-Immunology and Rheumatology Department, Hôpital NeckerEnfants Malades, Assistance Publique-Hôpitaux de Paris (AP-HP), Paris, France. 
${ }^{6}$ Paris Descartes University, Infectious Diseases and Tropical Medicine Department NeckerPasteur Infectious Diseases Center, Necker-Enfants Malades Hospital AP-HP, Paris, France.

${ }^{7}$ Paris Descartes University, Microbiology Department, Necker-Enfants Malades Hospital APHP, Paris, France

${ }^{8}$ INSERM UMR 1163, Paris, France, Imagine Institute, Paris, France.

${ }^{9}$ Paris Descartes University, Sorbonne Paris Cité, Imagine Institute, Paris, France.

10 Paris Descartes University, Paediatric Gastroenterology Hepatology and Nutrition Department, Hôpital Necker-Enfants Malades, AP-HP, Paris, France

${ }^{11}$ Study Center for Primary Immunodeficiencies, Necker-Enfants Malades University Hospital, Assistance Publique Hôpitaux de Paris (APHP), Paris, France.

${ }^{12}$ Paris Descartes University, Hematology Department, Necker-Enfants Malades University Hospital, Assistance Publique Hôpitaux de Paris (APHP), Paris, France.

13 Department of Orthopedic Pediatrics, Necker-Enfants Malades University Hospital, Assistance Publique Hôpitaux de Paris (APHP), Paris, France.

${ }^{14}$ Department of Pediatrics, Hôpital Jeanne de Flandre, University Hospital of Lille, Lille, France.

${ }^{15}$ Department of Clinical Immunology, Saint-Louis Hospital, Assistance Publique Hôpitaux de Paris (APHP), Paris, France.

${ }^{16}$ Collège de France, Paris, France.

${ }^{17}$ These authors contributed equally to this work. 
47 Institut Imagine

4824 Bld Montparnasse, 75015, Paris, France.

49 alain.fischer@aphp.fr

50

51 Professor Harry Sokol

52

Gastroenterology Department, Hôpital Saint-Antoine

53184 rue du Faubourg Saint-Antoine, 75571 Paris CEDEX 12, France

54 harry.sokol@aphp.fr

55

56 Word count: 3200

57

58

59 DECLARATION OF INTERESTS

60 The authors declare no competing financial interests.

61

62 
63 CAPSULE SUMMARY: The gut microbiota plays a key role in host physiology and is an 64 actor in inflammatory bowel disease pathogenesis. Patients with primary immunodeficiency 65 causing intestinal inflammation have disease-specific dysbiosis.

66

67 Keywords: gut microbiota; inflammatory bowel disease; primary immunodeficiency

68 
The gut microbiota is composed of a huge amount and diversity of microorganisms playing major roles in physiological and pathological settings such as inflammatory bowel disease (IBD). The triggering role of the gut microbiota on intestinal inflammation is known and an intestinal dysbiosis (i.e. an imbalance in the microbiota composition and function) has been pointed out in IBD 15 years ago. Moreover, a positive efficacy signal has been observed in studies evaluating fecal microbiota transplantation in IBD demonstrating that the gut microbiota alterations is an actor in the inflammatory process and not simple consequence ${ }^{1}$.

Environmental factors have an important effect on gut microbiota composition, but host genetic has an impact too. This effect has been demonstrated in knockout mouse for innate immunity genes. Microbiome genome-wide association studies have identified several associations between genetic polymorphisms and the gut microbiota composition offering some clues for the effects of genes on microbiota composition in humans ${ }^{2}$. However, the effect of major specific disease-causing genetic defects on gut microbiota has never been studied in humans yet.

Here we characterized the fecal microbiota composition of patients with three types of rare primary immunodeficiency (PID) causing IBD conditions, chronic granulomatous disease (CGD, 11 samples), X-linked inhibitor of apoptosis (XIAP, 7 samples) deficiency and partial Tetratricopeptide Repeat Domain 7A (TTC7A, 7 samples) deficiency, in comparison to patients with non-genetic determined IBD (18 samples) and healthy subjects (HS, 23 samples, Suppl Table 1).

The microbiota composition was assessed by $16 \mathrm{~S}$ sequencing. As seen previously, gut microbiota composition was influenced by age, antifungal and antibiotic treatment ${ }^{3}$ (Suppl 
Figure 1). Beta diversity analysis showed a remarkable clustering of samples according to disease phenotypes (Figure 1A). The difference with HS was statistically significant for CGD and TTC7A groups while it did not reach significance for XIAP (Figure 1 A-D). Interestingly the difference with IBD patients was statistically significant for all three PID patients groups (Figure 1A). Age was very heterogeneous in patients from TTC7A and XIAP groups (0.62 to 18 and 1.3 to 34 years respectively). The gut microbiota composition changes constantly between birth and the age of 3 years when it reaches an adult-like configuration ${ }^{4}$. We thus performed the same analysis after segregating the patients according to their age (younger or older than 3 years). The difference with HS was maintained for TTC7A patients in both age groups and reached significance in XIAP patients older than 3 (only 2 XIAP patients younger than 3) (Suppl Figure 2).

Patients with CGD, XIAP and TTC7A deficiency can experience intestinal inflammation mimicking $\mathrm{IBD}^{5}$. In our study population, samples were taken from patients with (PID $\mathrm{IBD}$ ) or without ( $\mathrm{PID}_{\text {no-IBD }}$ ) IBD involvement. In addition, PID patients could be in active phase (flare) or remission of IBD (PID, IBD flare/inactive). Microbiota from patients with active intestinal inflammation (PIDIBD (flare) ) was significantly different from the one of PID ${ }_{\text {no-IBD }}$ patients (Figure 1E). We observed a significant diversity reduction in patients with $\mathrm{PID}_{\mathrm{IBD}}$ compared to HS (Figure 1F, Suppl Figure 3A). Moreover, this reduction was even stronger in patients with active intestinal inflammation (Figure 1G, Suppl Figure 3B). These effects were seen in the three-studied PID although the numbers were too low to reach statistical significance when analyzing each PID independently (Suppl Figure 4).

In accordance with the published literature, the bacterial microbiota of all groups was dominated by bacteria from Firmicutes, Bacteroidetes, Actinobacteria and Proteobacteria phyla. Differences were noticed between PID patients and HS with notably a dramatic increase 
in Proteobacteria from the Enterobacteriaceae family in the TTC7A group and an increase proportion in bacteria from the Bacteroidetes phylum and from the Clostridiaceae family in the CGD and XIAP groups respectively (Figure $1 \mathrm{H}$, Suppl Figure 3C). These alterations were

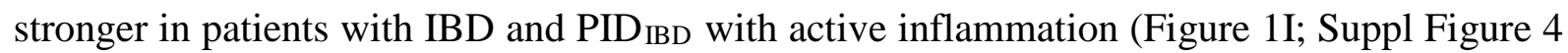
C, E). We then looked for the microbial features associated with the three studied PID and used a multivariate association test (MaAsLin) to control for potential confounding factors such as age, gender, smoking, intestinal inflammation and treatment. We observed several alterations specific to each PID compared to HS. Patients with TTC7A deficiency exhibited an increased abundance of Proteobacteria involving several components of the Gammaproteobacteria and Epsilonproteobacteria classes (Figure 2A). On the other hand, bacteria from the Ruminococcaceae family and notably the Oscillospira genera were decreased. Patients with XIAP deficiency exhibited an increased abundance of several bacterial taxa including members of the Proteobacteria, Firmicutes, Actinobacteria and Fusobacteria phyla (Figure 2B). Four of these taxa (Scardovia, Fusobacterium, Rothia dentocariosa, Veillonella) are not usually found in the gut but are known members of the oral microbiota that are implicated in dental caries, but also IBD, colorectal cancer, and liver diseases ${ }^{6}$. Interestingly, an increased abundance of oral bacteria in the gut has been observed in several intestinal and extra-intestinal diseases ${ }^{7}$. Moreover, a recent study showed that some members of the oral microbiota can have proinflammatory effects when colonizing the gut ${ }^{7}$ suggesting a possible direct effect of these oral bacteria in the intestinal inflammatory phenotype observed in patients with XIAP deficiency. Another important specificity in XIAP patients was the presence of Lactococcus garvieae which is a highly virulent pathogen affecting saltwater fish ${ }^{8}$ and is rarely involved in human infection. L. garvieae was found at a high level (0.23-0.5\% of all reads) in XIAP patients with active intestinal inflammation but not in all other patients in this study. Patients with CGD 
exhibited an increased abundance of Ruminococcus gnavus that has also been associated with ileal Crohn’s disease ${ }^{9}$ (Figure 2C).

In summary, we showed that the gut microbiota of patients with CGD, TTC7A and XIAP deficiency have distinct alterations suggesting a primary defect in host immune system as a basis of dysbiosis. Although it remains to be experimentally documented, the microbial alterations induced by the host genetic defect might play a role in some aspects of the PID phenotype and particularly intestinal involvement. The number of subjects studied was low because of the extreme rarity of the studied diseases. However, independent and ideally larger and longitudinal studies are required to confirm our findings. Controlling for potential confounding factors such as diet, treatment, age, delivery mode, socio-economic features, gastrointestinal symptoms, is particularly difficult in studies aiming at discriminating genetic factors from environmental and inflammation influences. Similarly, identification of ideal controls is not trivial. Although non-affected healthy siblings sharing similar lifestyles would be attractive, there are obvious limitations in term of feasibility.

Finally, if these observations are confirmed, the alteration in gut microbiota composition might have clinical interest as diagnosis biomarkers.

Harry Sokol ${ }^{1,2,3,4}$, Nizar Mahlaoui ${ }^{4,5,17}$, Claire Aguilar ${ }^{6,8,17}$, Perrine Bach ${ }^{4}$, Olivier JoinLambert $^{7}$, Aurélie Garraffo, ${ }^{6}$, Philippe Seksik ${ }^{1,3}$, François Danion ${ }^{6}$, Sarah Jegou ${ }^{1}$, Marjolene Straube ${ }^{2}$, Christelle Lenoir ${ }^{8}$, Bénédicte Neven ${ }^{5,8,9}$, Despina Moshous ${ }^{5,8,9}$, Stéphane Blanche, Bénédicte Pigneur ${ }^{8,10}$, Olivier Goulet ${ }^{8,10}$, Frank Ruemmele ${ }^{8,10}$, Felipe Suarez ${ }^{4,8,12}$, Laurent Beaugerie $^{3}$, Stéphanie Pannier ${ }^{11,13}$, Françoise Mazingue ${ }^{14}$, Olivier Lortholary ${ }^{4,6,9}$, Lionel 
Galicier $^{15}$, Capucine Picard ${ }^{8,9,11,4}$, Geneviève de Saint Basile $8,9,11$, Sylvain Latour ${ }^{8,9}$, Alain Fischer $^{4,5,8,9,16}$

${ }^{1}$ Sorbonne Universités, UPMC Univ. Paris 06, École normale supérieure, PSL Research University, CNRS, INSERM, APHP, Laboratoire des Biomolécules (LBM), 27 rue de Chaligny, 75005 Paris, France

${ }^{2}$ INRA, UMR1319 Micalis \& AgroParisTech, Jouy en Josas, France

${ }^{3}$ Department of Gastroenterology, Saint Antoine Hospital, AP-HP, UPMC Univ Paris 06, Paris, France

${ }^{4}$ French National Reference Center for Primary Immune Deficiency (CEREDIH), Necker Enfants Malades University Hospital, Assistance Publique-Hôpitaux de Paris (APHP), Paris, France

5 Paediatric Haematology-Immunology and Rheumatology Department, Hôpital NeckerEnfants Malades, Assistance Publique-Hôpitaux de Paris (AP-HP), Paris, France.

${ }^{6}$ Paris Descartes University, Infectious Diseases and Tropical Medicine Department NeckerPasteur Infectious Diseases Center, Necker-Enfants Malades Hospital AP-HP, Paris, France.

${ }^{7}$ Paris Descartes University, Microbiology Department, Necker-Enfants Malades Hospital APHP, Paris, France

${ }^{8}$ INSERM UMR 1163, Paris, France, Imagine Institute, Paris, France.

${ }^{9}$ Paris Descartes University, Sorbonne Paris Cité, Imagine Institute, Paris, France.

10 Paris Descartes University, Paediatric Gastroenterology Hepatology and Nutrition Department, Hôpital Necker-Enfants Malades, AP-HP, Paris, France 
${ }^{11}$ Study Center for Primary Immunodeficiencies, Necker-Enfants Malades University Hospital, 189 Assistance Publique Hôpitaux de Paris (APHP), Paris, France.

$190{ }^{12}$ Paris Descartes University, Hematology Department, Necker-Enfants Malades University 191 Hospital, Assistance Publique Hôpitaux de Paris (APHP), Paris, France.

19213 Department of Orthopedic Pediatrics, Necker-Enfants Malades University Hospital, 193 Assistance Publique Hôpitaux de Paris (APHP), Paris, France.

$194{ }^{14}$ Department of Pediatrics, Hôpital Jeanne de Flandre, University Hospital of Lille, Lille, 195 France.

$196{ }^{15}$ Department of Clinical Immunology, Saint-Louis Hospital, Assistance Publique Hôpitaux de 197 Paris (APHP), Paris, France.

$198 \quad{ }^{16}$ Collège de France, Paris, France.

$199{ }^{17}$ These authors contributed equally to this work. 
Financial support by patient association Association François Aupetit (SL, CA), ANR- 08MIEN-012-01 (SL).

\section{AUTHOR CONTRIBUTION}

205

Recruited patients with PID: Alain Fischer, Nizar Mahlaoui, Perrine Bach, Bénédicte Neven, Despina Moshous, Stéphane Blanche, Francoise Mazingue, Lionel Galicier, Claire Aguilar, Aurélie Garraffo, François Danion, Olivier Lortholary, Felipe Suarez.

Recruited patients with IBD: Harry Sokol, Laurent Beaugerie, Philippe Seksik, Olivier Goulet, Frank Ruemmele, Benedicte Pigneur.

Recruited controls: Stéphanie Pannier, Perrine Bach, Francois Danion

Genetic diagnosis of patients with PID: Geneviève de Saint Basile, Capucine Picard,Sylvain Latour, Christelle Lenoir

Original study developed by Sylvain Latour, Claire Aguilar and Alain Fischer

Analysis of the data and manuscript writing: Harry Sokol, Alain Fischer, Nizar Mahlaoui.

\section{REFERENCES}

1. Pigneur B, Sokol H. Fecal microbiota transplantation in inflammatory bowel disease: the quest for the holy grail. Mucosal Immunol. 2016;9:1360-5.

2. Hall AB, Tolonen AC, Xavier RJ. Human genetic variation and the gut microbiome in disease. Nat Rev Genet. 2017;18:690-9.

3. Morgan XC, Tickle TL, Sokol H, Gevers D, Devaney KL, Ward DV, et al. Dysfunction of the intestinal microbiome in inflammatory bowel disease and treatment. Genome Biol. 2012;13:R79. 
4. Tamburini S, Shen N, Wu HC, Clemente JC. The microbiome in early life: implications for health outcomes. Nat Med. 2016;22:713-22.

5. Uhlig HH. Monogenic diseases associated with intestinal inflammation: implications for the understanding of inflammatory bowel disease. Gut. 2013;62:1795-805.

6. Kummen M, Holm K, Anmarkrud JA, Nygård S, Vesterhus M, Høivik ML, et al. The gut microbial profile in patients with primary sclerosing cholangitis is distinct from patients with ulcerative colitis without biliary disease and healthy controls. Gut. 2017;66:611-9.

7. Atarashi K, Suda W, Luo C, Kawaguchi T, Motoo I, Narushima S, et al. Ectopic colonization of oral bacteria in the intestine drives TH1 cell induction and inflammation. Science. 2017;358:359-365.

8. Vendrell D, Balcázar JL, Ruiz-Zarzuela I, de Blas I, Gironés O, Múzquiz JL. Lactococcus garvieae in fish: a review. Comp Immunol Microbiol Infect Dis. 2006;29:177-98.

9. Sokol H, Leducq V, Aschard H, Pham H-P, Jegou S, Landman C, et al. Fungal microbiota dysbiosis in IBD. Gut. 2016;

\section{FIGURE LEGENDS}

Figure 1: Abnormal bacterial microbiota composition and diversity in PID. Principal coordinate analysis of Bray-Curtis distance with each sample colored according to the studied group. PC1, PC2 and PC3 represent the top three principal coordinates that captured most of the diversity. The fraction of diversity captured by the coordinate is given as a percentage. Groups were compared using ANOSIM method (9999 permutations). (A) All the studied groups plotted together. (B) CGD group compared to healthy subjects. (C) TTC7A group compared to healthy subjects. (D) XIAP group compared to healthy subjects. (E) Patients with active IBD involvement (PID $\mathrm{IBD}$ (flare) $)$ compared to non-active IBD involvement ( $\mathrm{PID}$ IBD(nonactive) $)$ and without IBD involvement ( $\left.\mathrm{PID}_{\text {no-IBD }}\right) .(\mathbf{F}, \mathbf{G})$ Bacterial diversity based on the Shannon index in the fecal samples of indicated groups. Statistical significance was assessed using ANOVA with a post hoc Dunn's test. (H,I) Global composition of bacterial microbiota at the phylum levels for the indicated groups *: $\mathrm{p}<0.05 ;{ }^{* *}$ : $\mathrm{p}<0.01$; ${ }^{* * *}: \mathrm{p}<0.001 ; * * * *: \mathrm{p}<0.0001$. 
256 Figure 2: Bacterial taxa associated with TTC7A, XIAP and CGD. Bacterial taxa associated 257 with TTC7A deficiency (A), XIAP deficiency (B) and CGD (C). Differences in abundance are 258 shown for the bacterial taxa detected using a multivariate statistical approach (see 'Material and 259 Methods'). All the indicated differences are statistically significant with $\mathrm{p}<0.05$ after correction 260 for multiple testing. The fold change for each taxon was calculated by dividing the mean 261 abundance in the cases by that of the controls. 
Figure 1

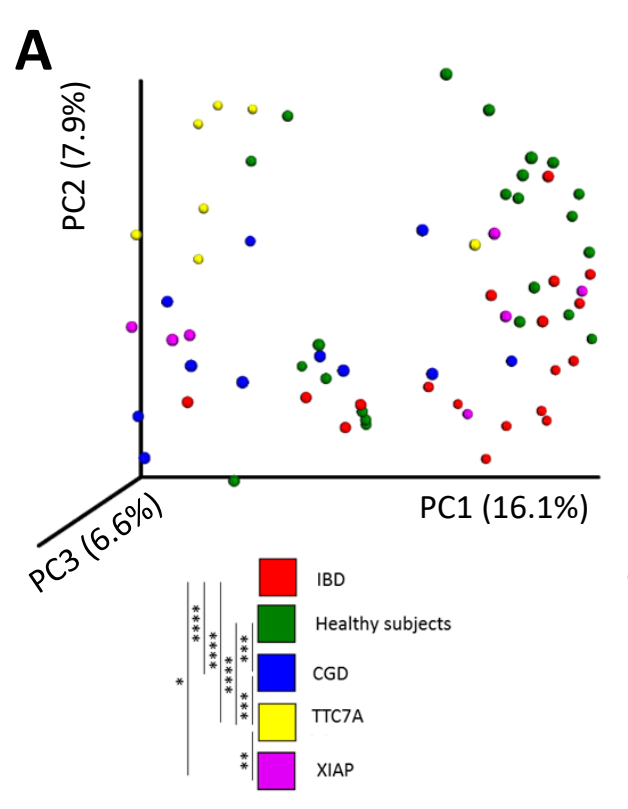

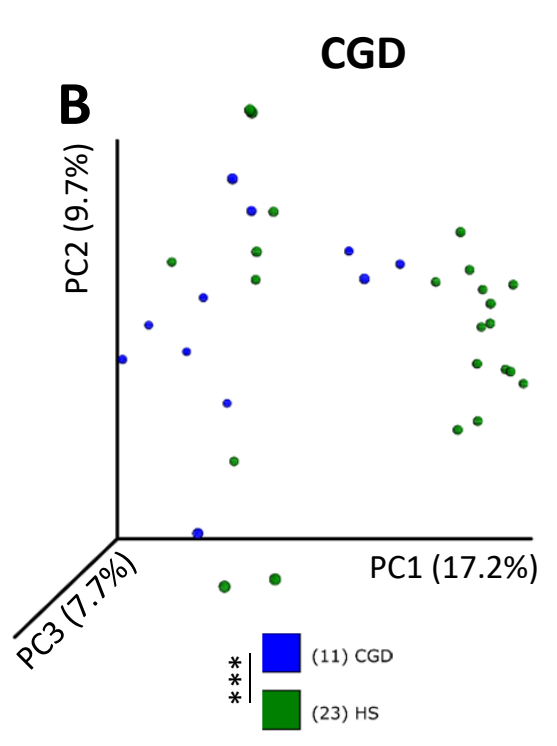
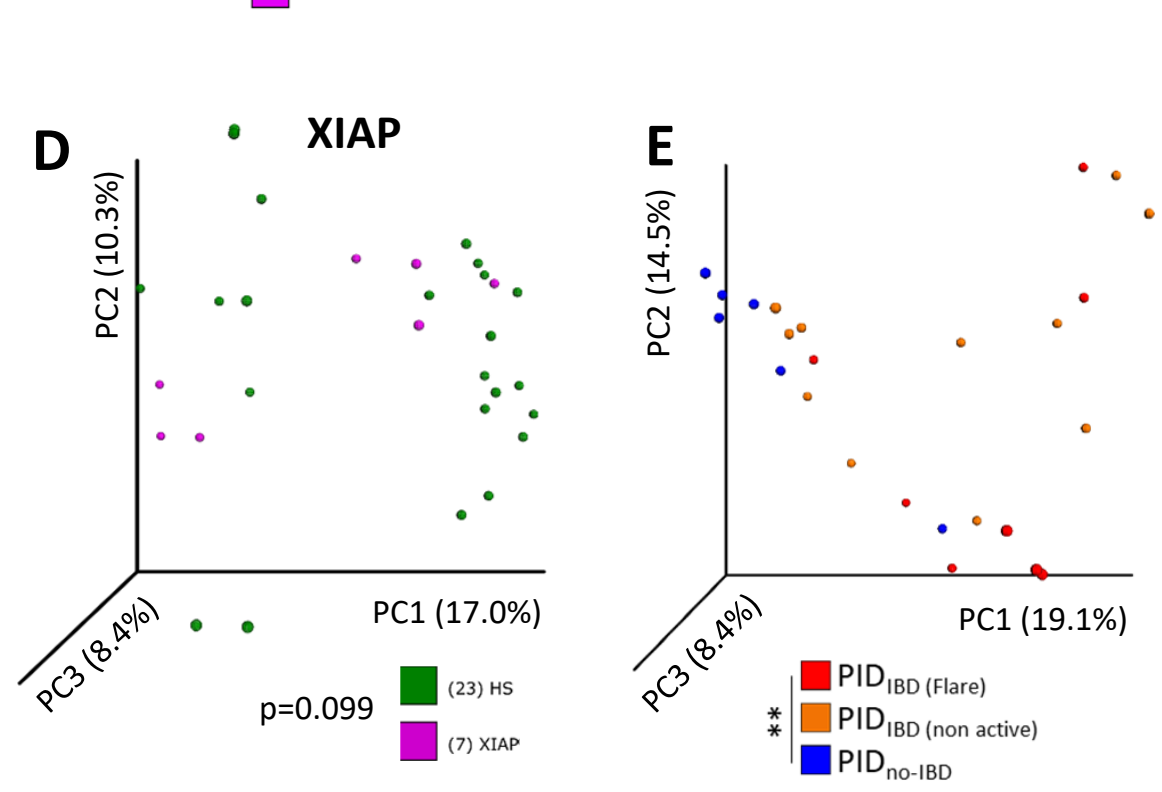

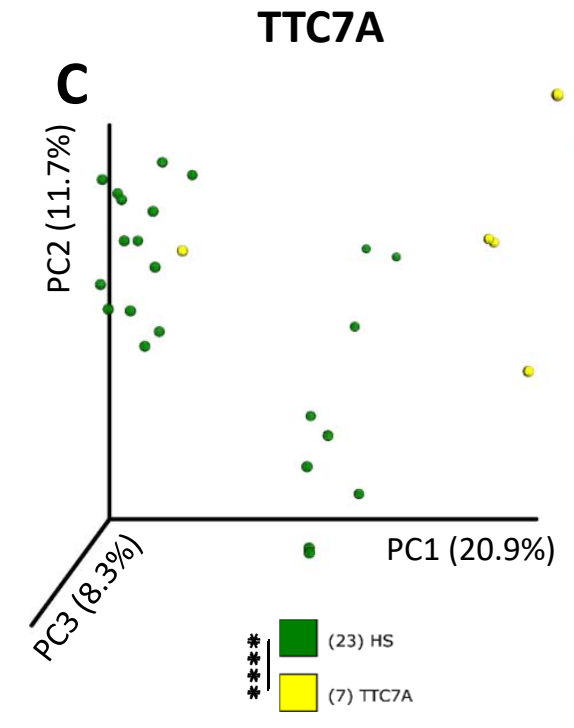

F
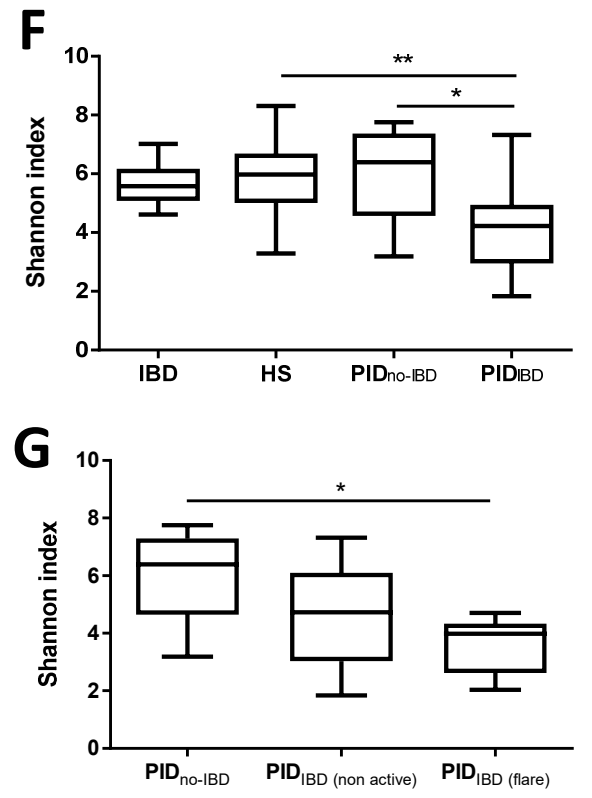

Phylum
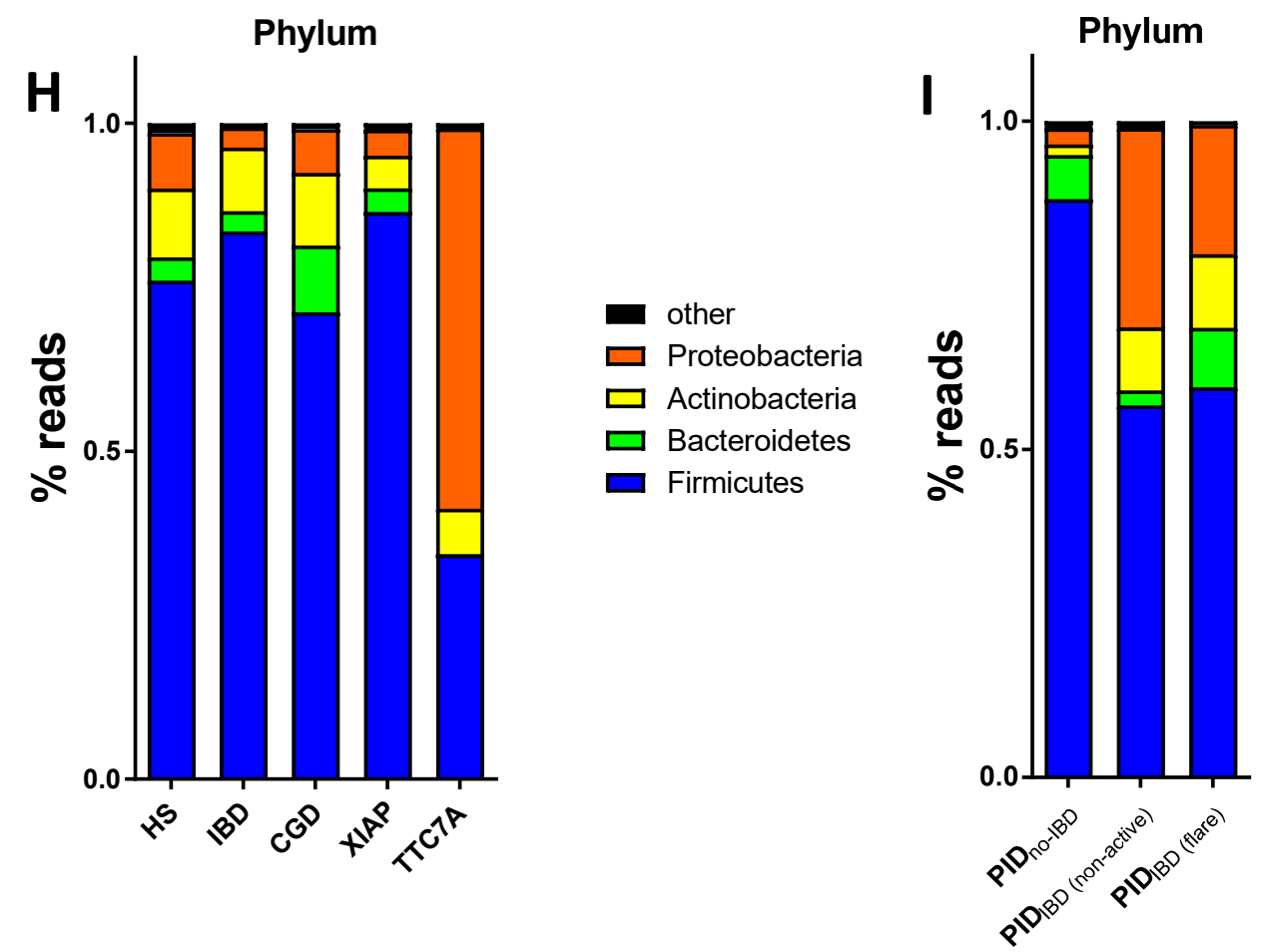

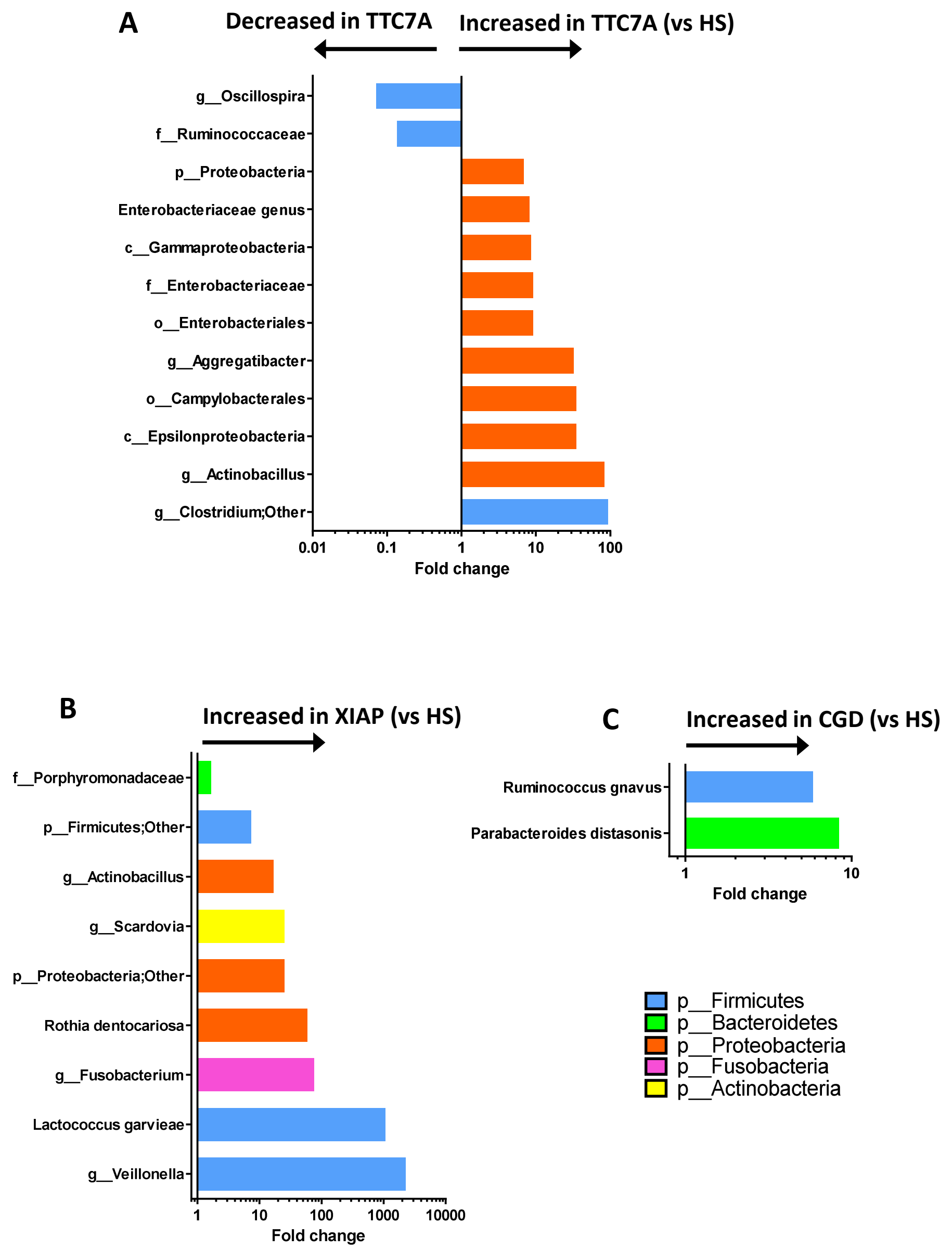
Effect of age (Bray curtis)

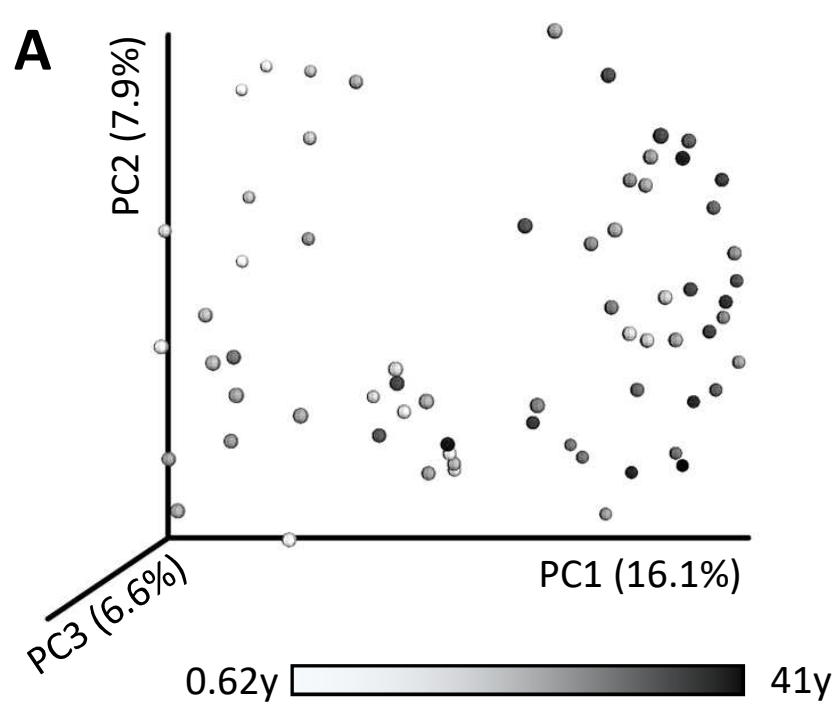

Effect of antifungical treatment (Bray curtis)

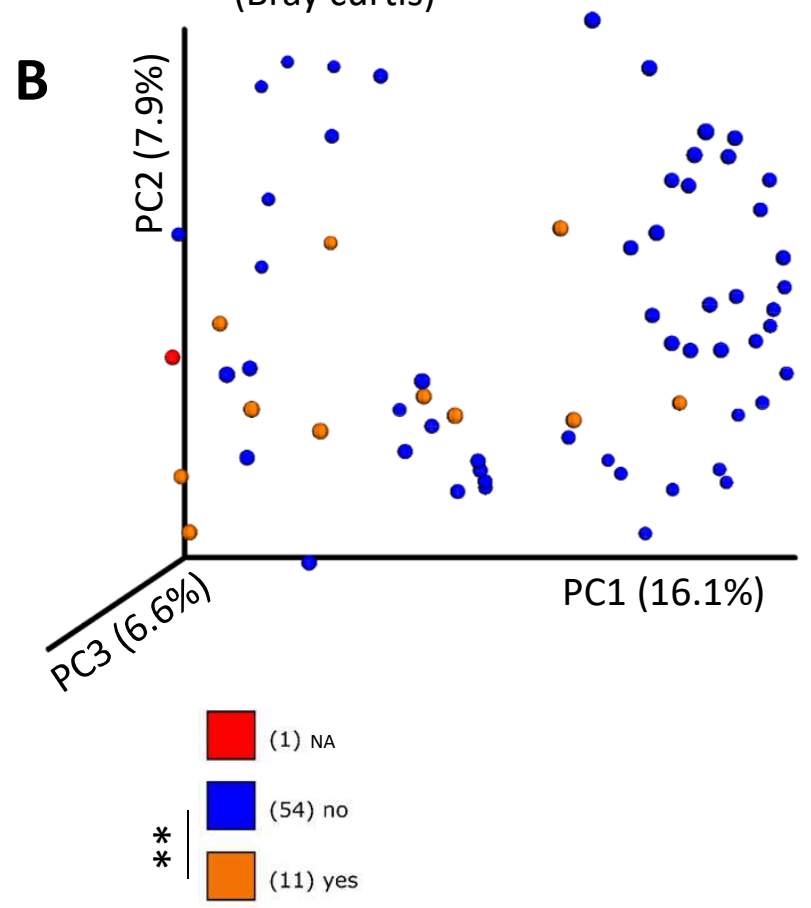

Effect of antibiotic treatment (Bray curtis)

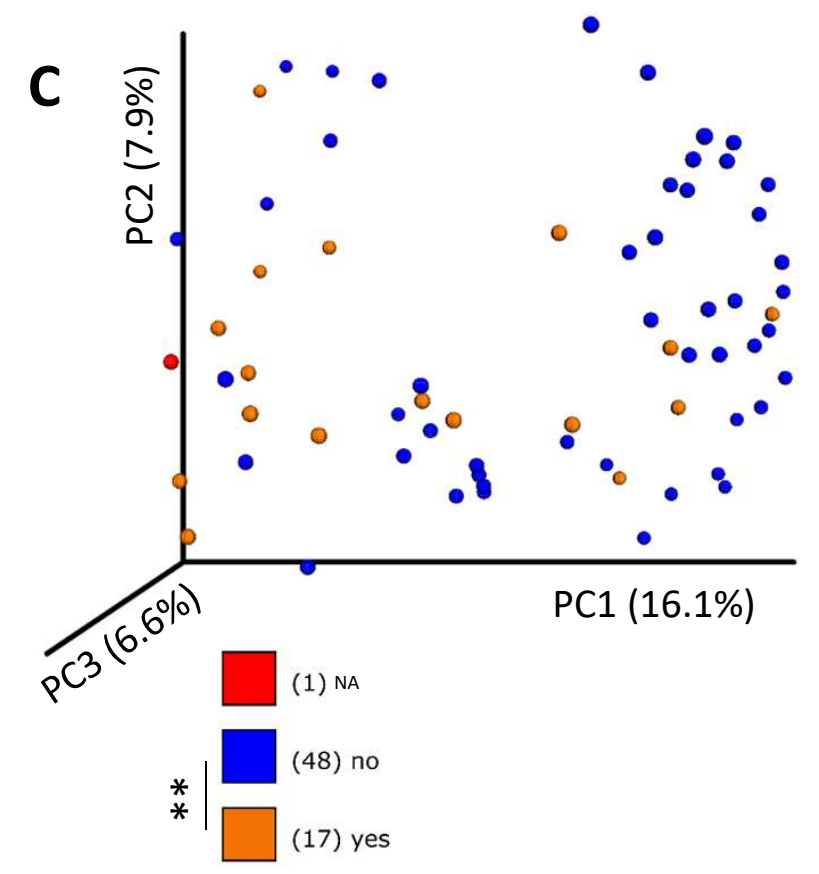


Supplementary Figure 2

$<3$ years

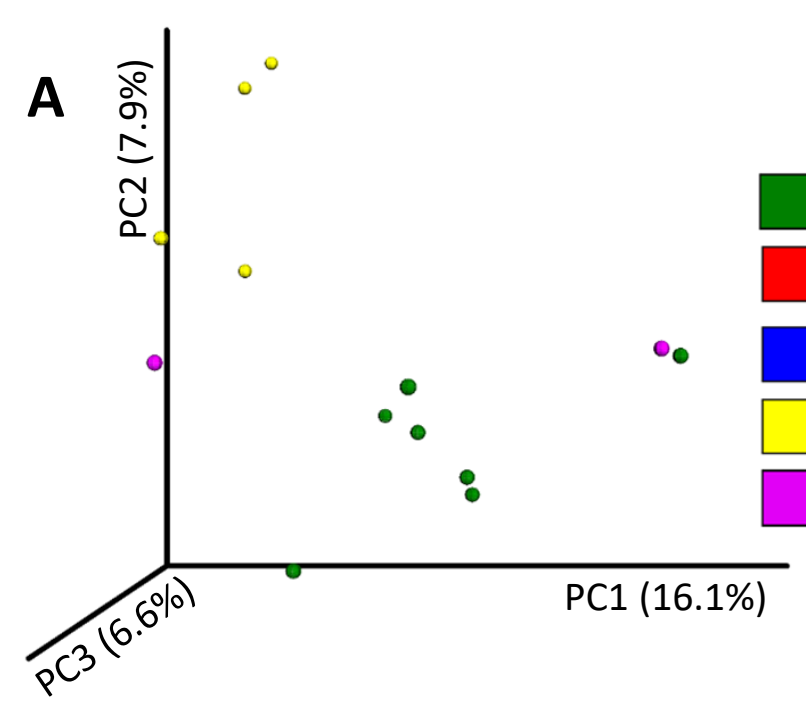

C
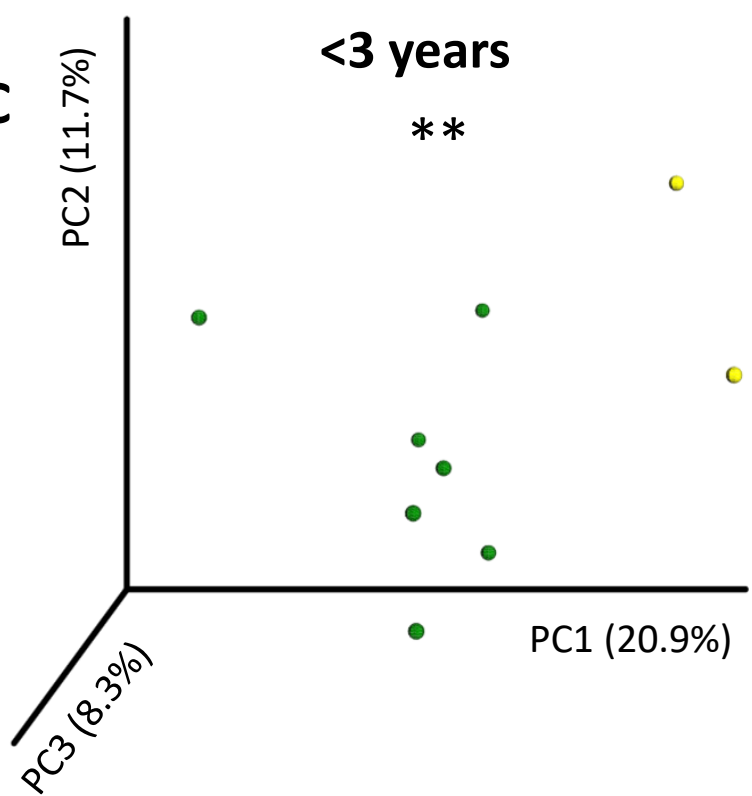

- <3 years

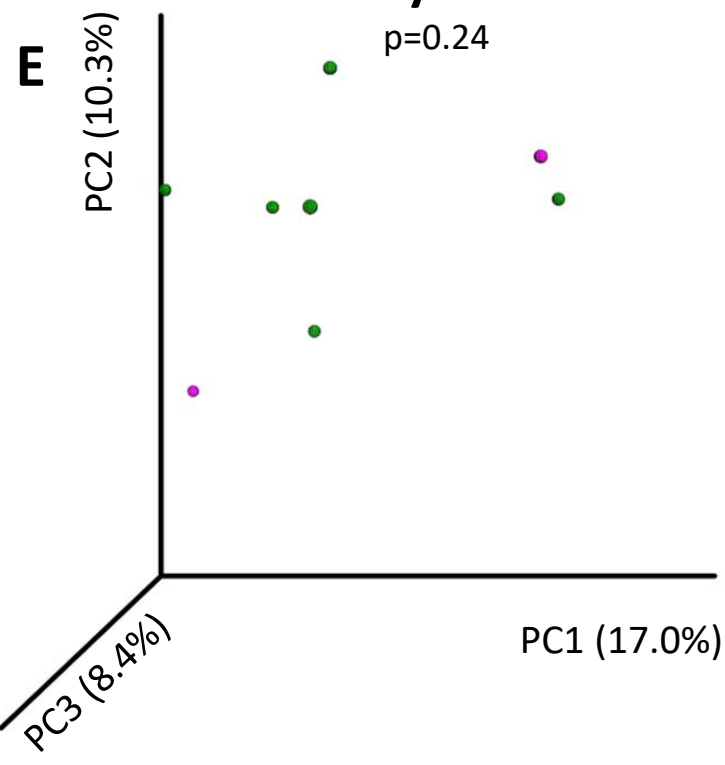

$>3$ years

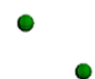

Healthy subjects

IBD

CGD

TTC7A

XIAP
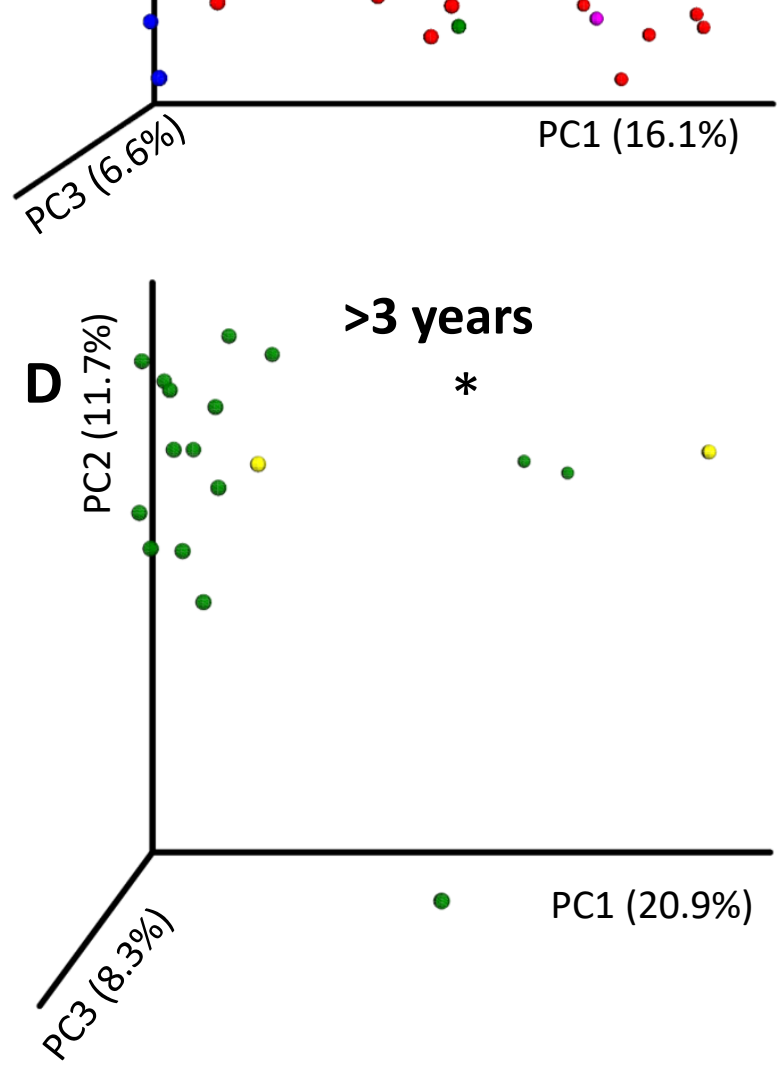

- >3 years

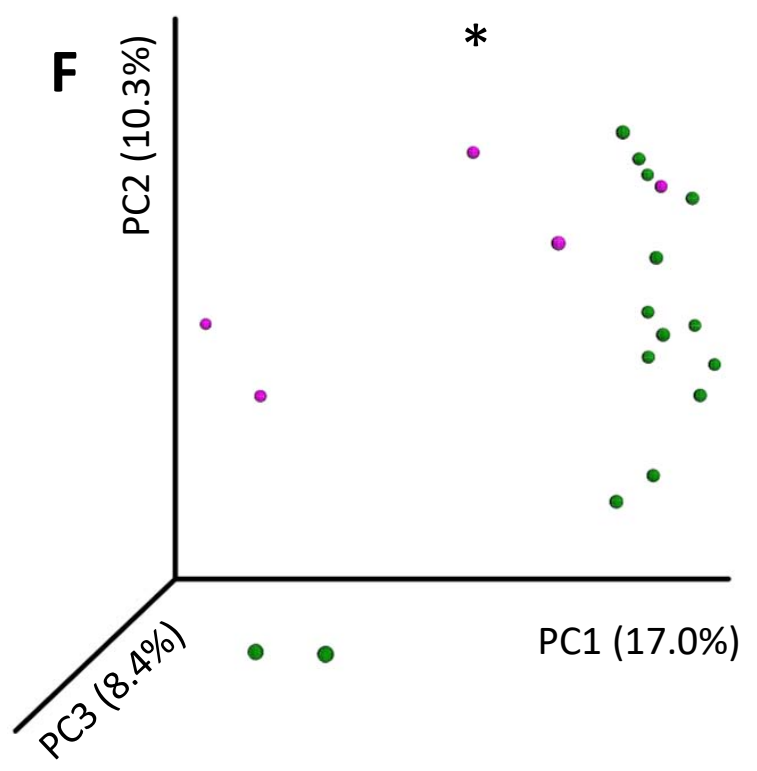




\section{Supplementary Figure 3}

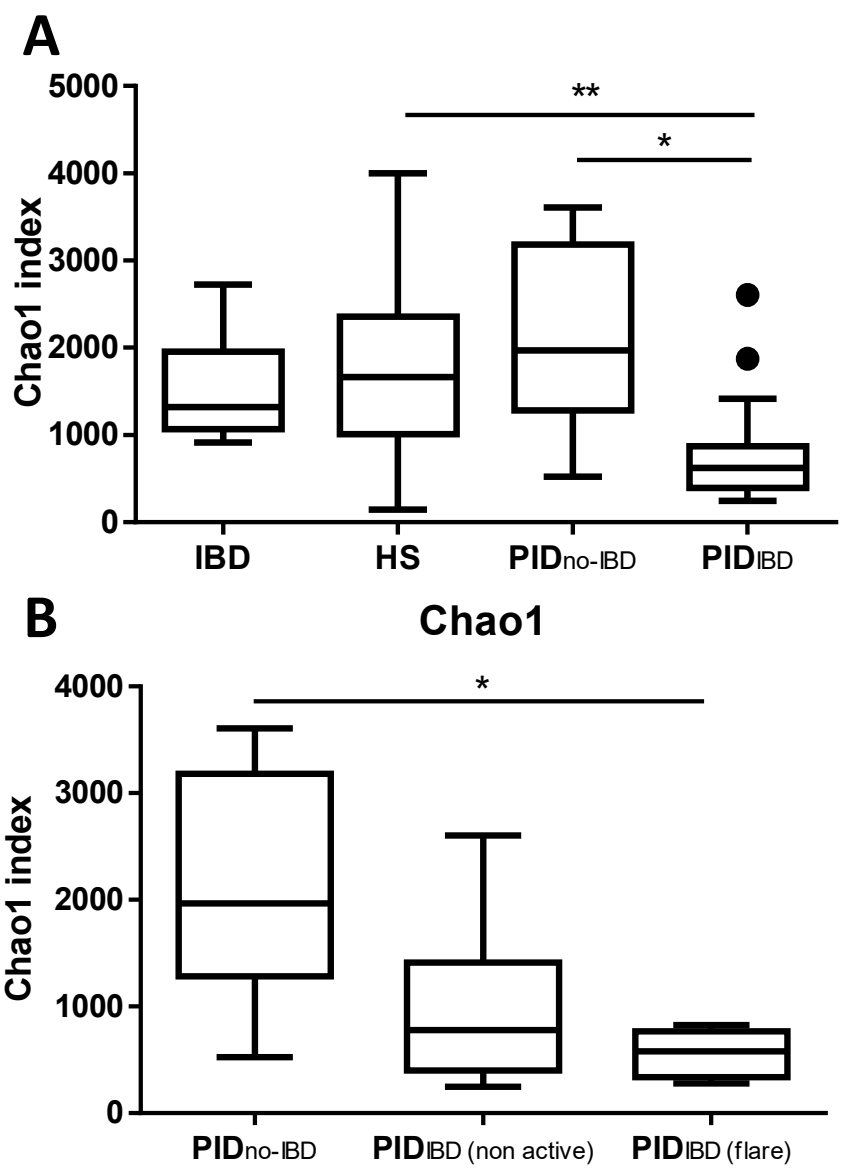

Family
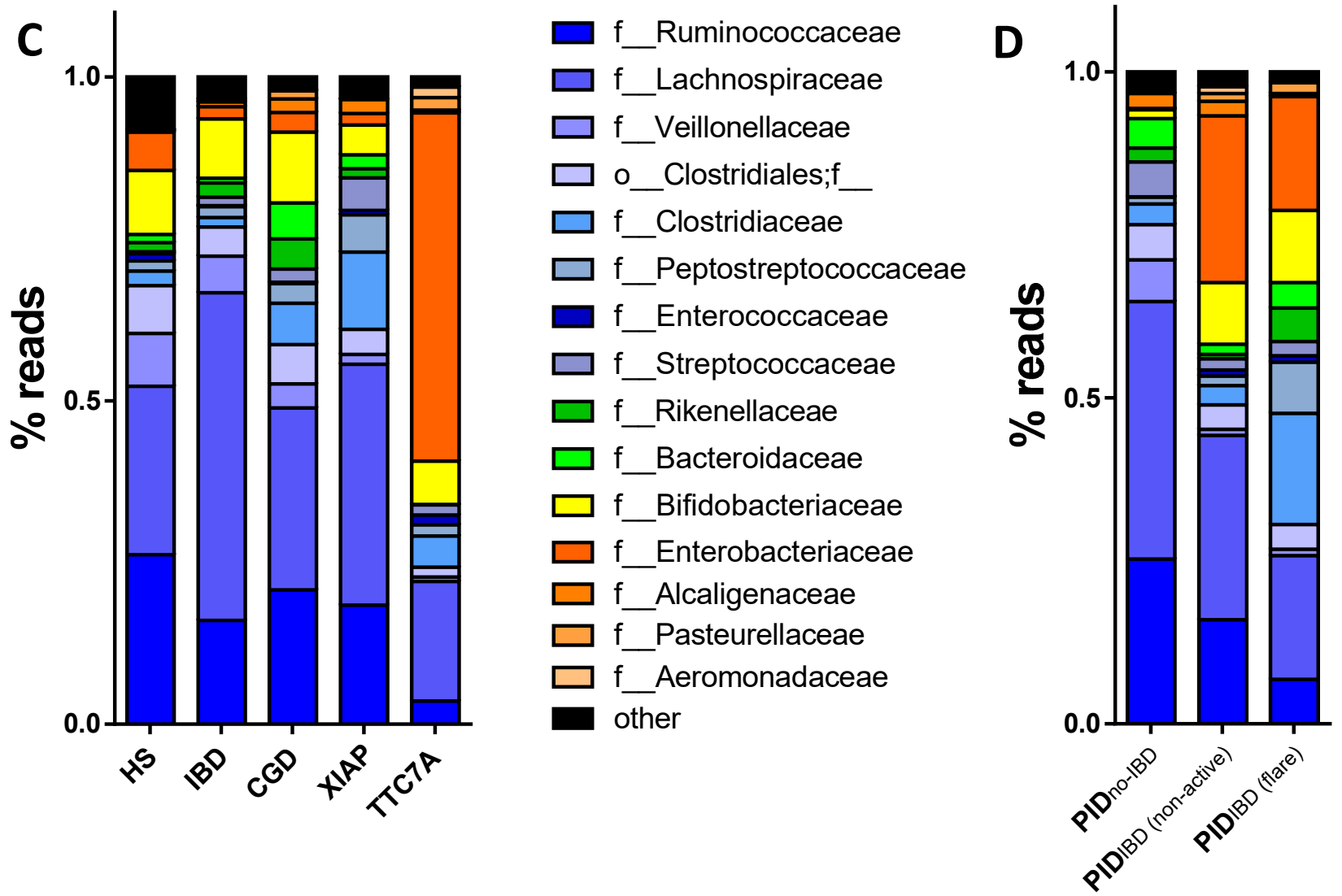

Family 

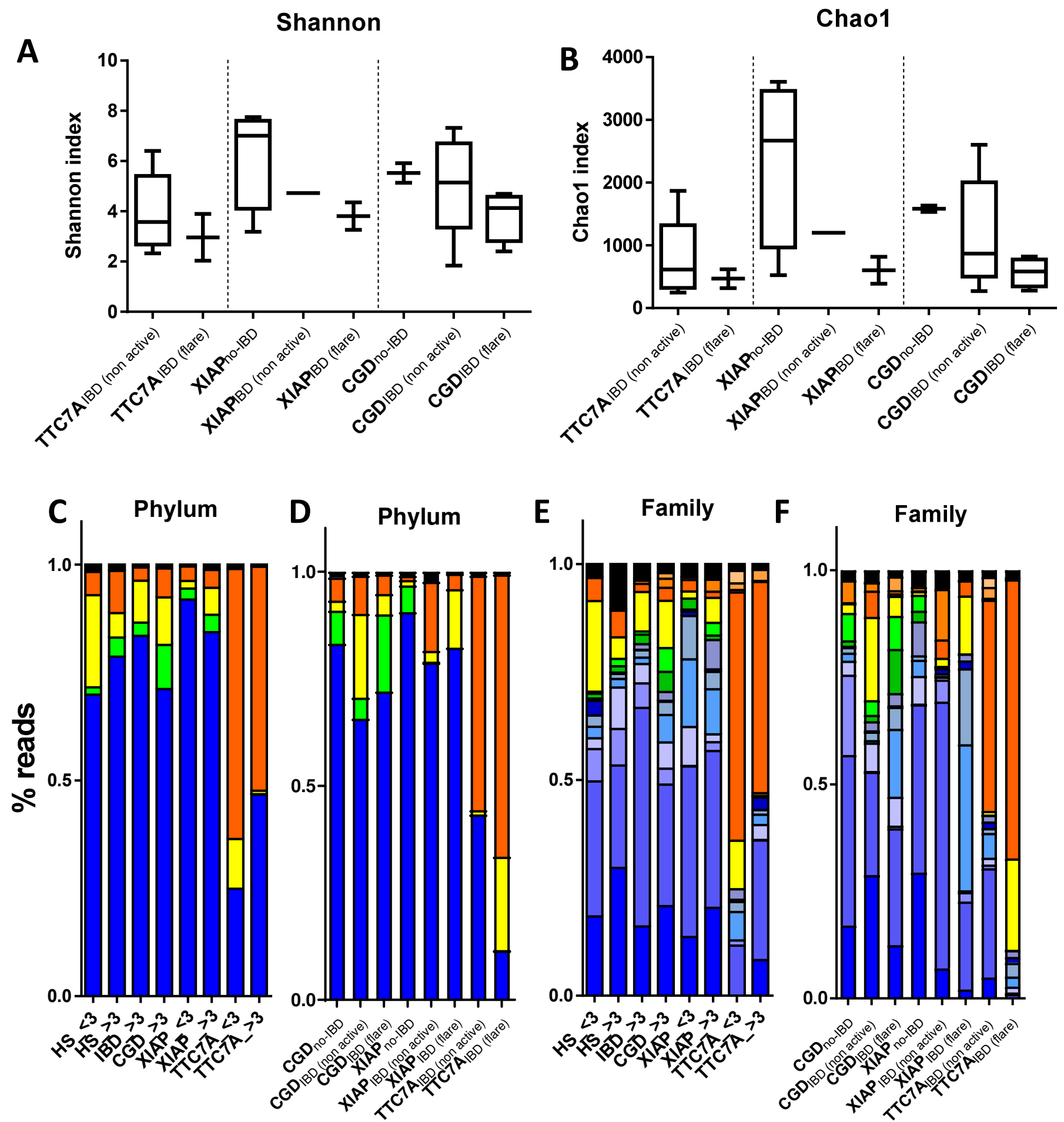

other

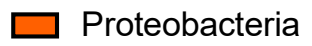

$\square$ Actinobacteria

$\square$ Bacteroidetes

$\square$ Firmicutes
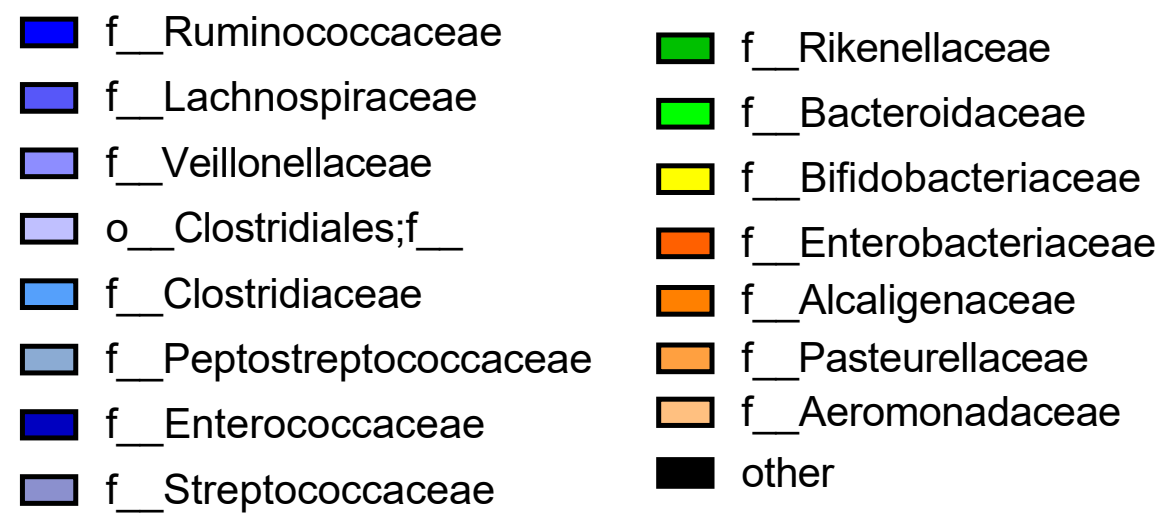
Supplementary Table 1 : Characteristics of patients

\begin{tabular}{|c|c|c|c|c|c|}
\hline Patients $\mathrm{n}$ & $\begin{array}{l}\text { HS } \\
23\end{array}$ & $\begin{array}{c}\text { IBD } \\
18\end{array}$ & $\begin{array}{c}\text { CGD } \\
10\end{array}$ & $\begin{array}{c}\text { TTC7A } \\
5\end{array}$ & $\begin{array}{c}\text { XIAP } \\
6\end{array}$ \\
\hline Sample $n$ & 23 & 18 & 11 & 7 & 7 \\
\hline Age: median (min-max) & $9(0.83-32)$ & $28.5(10-41)$ & $10(6-31)$ & $1.8(0.62-18)$ & $8(1.3-34)$ \\
\hline Male Gender \% ( $n$ ) & $61 \%(14)$ & $89 \%(16)$ & $100 \%(11)$ & $43 \%(3)$ & $100 \%(7)$ \\
\hline Gut inflammation history & 0 & $100 \%(18)$ & $82 \%(9)$ & $100 \%(7)$ & $43 \%(3)$ \\
\hline Active gut inflammation & 0 & 0 & $64 \%(7)$ & $71 \%(5)$ & $71 \%(5)$ \\
\hline Smoking & $4 \%(1)$ & $22 \%(4)$ & 0 & 0 & $43 \%(3)$ \\
\hline $\begin{array}{l}\text { Sulfamethoxazole / } \\
\text { trimethoprim }\end{array}$ & 0 & 0 & $100 \%(11)$ & $29 \%(2)$ & $50 \%(3)$ \\
\hline Other antibiotics & 0 & 0 & $27 \%(3)$ & 0 & $29 \%(2)$ \\
\hline Itraconazole & 0 & 0 & $91 \%(10)$ & 0 & 0 \\
\hline Posaconazole & 0 & 0 & $9 \%(1)$ & 0 & 0 \\
\hline 5-ASA & 0 & $33 \%(6)$ & $18.2 \%(2)$ & 0 & 0 \\
\hline Corticosteroid & 0 & 0 & 0 & 0 & $33 \%(2)$ \\
\hline Azathioprin & 0 & $50 \%(9)$ & 0 & 0 & 0 \\
\hline Methotrexate & 0 & 0 & $9 \%(1)$ & 0 & $17 \%(1)$ \\
\hline Anti-TNF $\alpha$ & 0 & $61 \%(11)$ & 0 & 0 & 0 \\
\hline Anti IL1 therapy & 0 & 0 & 0 & 0 & $17 \%(1)$ \\
\hline $\begin{array}{l}\text { Natalizumab } \\
\text { (anti } \alpha 4) \text { therapy }\end{array}$ & 0 & 0 & 0 & 0 & $17 \%(1)$ \\
\hline Any immunosuppressant & 0 & $61 \%(11)$ & $9 \%(1)$ & 0 & $50 \%(3)$ \\
\hline PPI & 0 & 0 & 0 & $14 \%(1)$ & 0 \\
\hline Ig substitution & 0 & 0 & 0 & $100 \%(7)$ & $17 \%(1)$ \\
\hline Enteral nutrition & 0 & 0 & 0 & $43 \%(3)$ & 0 \\
\hline Hydroxychloroquine & 0 & 0 & $9 \%(1)$ & 0 & 0 \\
\hline
\end{tabular}

5-ASA: 5 aminosaclicylate ; PPI: proton pump inhibitor ; Ig: Immunoglobulin substitution

1 CGD patient, 1 XIAP patient and 2 TTC7A patients experienced flare during the follow up and were then sampled twice 


\section{SUPPLEMENTARY INFORMATION}

\section{MATERIAL AND METHODS}

\section{Patients and sample collection}

Patients with primary immunodeficiency were recruited at Necker-Enfants malades University Hospital, Paris, France (Pediatric Immunology-Hematology-Rheumatology Unit, Adult Hematology Unit, Adult infectious Disease Unit), Saint-Louis Hospital, Paris, France and Lille Regional University Hospital, Lille, France and provided informed consent. Pediatric healthy subjects were recruited from the pediatric orthopedic surgery unit, Necker-Enfants malades University Hospital. Approval was obtained from the local ethics committee (Comité de Protection des Personnes III (Ref.: 3149, 2014, June $10^{\text {th }}$, Dipobiota study, ClinicalTrials.gov Identifier: NCT02909244). Patients with IBD in remission were recruited at the Gastroenterology Department of the Saint Antoine Hospital (Paris, France) and provided informed consent (local ethics committee: Comité de Protection des Personnes Ile-de-France IV, Suivitheque study). None of the IBD patients and the healthy subjects reported having taken antibiotics, probiotics or using colon-cleansing products for at least 1 months prior to enrollment. Patient characteristics are presented in Table 1. Whole stools were collected in sterile boxes and immediately homogenized, and $0.2 \mathrm{~g}$ aliquots were frozen at $-80^{\circ} \mathrm{C}$ for further analysis. Patients' data were collected, including clinical, treatment, immunological and genetic diagnosis. Enrolled patients with PID had damaging causal mutation respectively in either the TTC7A gene "ELA syndrome" ${ }^{26}$, BIRC4 (XIAP deficiency) and CYBBNox2 (X-linked CGD). None of the subjects received probiotics.

\section{Fecal DNA extraction}

Genomic DNA was extracted from $200 \mathrm{mg}$ of feces as previously described. ${ }^{27}$. Following microbial lysis with both mechanical and chemical steps, nucleic acids were precipitated in isopropanol for 10 minutes at room temperature, incubated for 15 minutes on ice and 
centrifuged for 30 minutes at $15,000 \mathrm{~g}$ and $4^{\circ} \mathrm{C}$. Pellets were suspended in $112 \mu \mathrm{L}$ of phosphate buffer and $12 \mu \mathrm{L}$ of potassium acetate. After RNase treatment and DNA precipitation, nucleic acids were recovered via centrifugation at $15,000 \mathrm{~g}$ and $4^{\circ} \mathrm{C}$ for 30 minutes. The DNA pellet was suspended in $100 \mu \mathrm{L}$ of TE buffer.

\section{S rRNA gene sequencing}

Fecal DNA was extracted from the weighted feces before and during the infection as previously described $^{27}$. Microbial diversity was determined for each sample by targeting a portion of the ribosomal genes. A 16S rRNA gene fragment comprising V3 and V4 hypervariable regions (16S; 5'-TACGGRAGGCAGCAG-3' and 5'-CTACCNGGGTATCTAAT-3') was amplified using an optimized and standardized 16S-amplicon-library preparation protocol (Metabiote, GenoScreen). Briefly, 16S rRNA gene PCR was performed using 5 ng genomic DNA according to the manufacturer's protocol (Metabiote) using 192 bar-coded primers (Metabiote MiSeq Primers, GenoScreen) at final concentrations of $0.2 \mu \mathrm{M}$ and an annealing temperature of $50^{\circ} \mathrm{C}$ for 30 cycles. The PCR products were purified using an Agencourt AMPure XP-PCR Purification system (Beckman Coulter), quantified according to the manufacturer's protocol, and multiplexed at equal concentrations. Sequencing was performed using a 250-bp paired-end sequencing protocol on an Illumina MiSeq platform (Illumina) at GenoScreen. Raw paired-end reads were subjected to the following process: (1) quality filtering using the PRINSEQ-lite PERL script ${ }^{5}$ by truncating the bases from the $3^{\prime}$ end that did not exhibit a quality $<30$ based on the Phred algorithm; (2) paired-end read assembly using FLASH (fast length adjustment of short reads to improve genome assemblies) with a minimum overlap of 30 bases and a $97 \%$ overlap identity; and (3) searching and removing both forward and reverse primer sequences using CutAdapt, with no mismatches allowed in the primers sequences. Assembled sequences for which perfect forward and reverse primers were not found were eliminated.

\section{S rRNA gene sequence analysis}


The sequences were demultiplexed and quality filtered using the Quantitative Insights Into Microbial Ecology (QIIME, version 1.9.1) software package ${ }^{28}$, and the forward and reverse Illumina reads were joined using the fastq-join method (http://code.google.com/p/ea-utils). The sequences were assigned to OTUs using the UCLUST algorithm ${ }^{29}$ with a $97 \%$ threshold of pairwise identity and classified taxonomically using the Greengenes reference database ${ }^{30}$. Principal component analyses of the Bray Curtis distance were built and used to assess the variation between experimental groups (beta diversity). Significance was assessed using ANOSIM (9999 permutations). The Shannon and Chao1 diversity index were calculated using rarefied data (depth $=17,000$ sequences/sample) and used to characterize species diversity in a community.

\section{Statistical analysis}

GraphPad Prism version 6.0 (San Diego, CA) was used for all analyses and graph preparation. For all graph data, the results are expressed as the mean \pm SEM, and statistical analyses were performed using the 2-tailed nonparametric Mann-Whitney $U$-test or Kruskal-Wallis test with Dunn’s Multiple Comparison Test. Statistical significance of sample grouping for beta diversity analysis was performed using Anosim method (9999 permutations). Differences with a p value less than 0.05 were considered significant.

Multivariate Analysis by Linear Models (MaAsLin), a multivariate statistical framework, was used to find associations between clinical metadata and microbial community abundance ${ }^{31}$. We took into account as much factor as possible in the analysis to control for the effects of potential confounding factors including: age, gender, smoking, intestinal inflammation and treatment and enteral nutrition. 


\section{SUPPLEMENTARY FIGURE LEGEND}

\section{Supplementary Figure 1: Beta diversity analysis reveals effect of age, antibiotics and}

antifungics on the microbiota composition. Principal coordinate analysis of Bray-Curtis distance with each sample colored according to the studied group. PC1, PC2 and PC3 represent the top three principal coordinates that captured most of the diversity. The fraction of diversity captured by the coordinate is given as a percentage. Groups were compared using ANOSIM method (9999 permutations). All the studied samples are plotted together and colored according to age (A), antifungic treatment $(\mathbf{B})$, and antibiotic treatment $(\mathbf{C}){ }^{*}: \mathrm{p}<0.05$; ${ }^{* *}$ : $\mathrm{p}<0.01 ; * * *$ : $\mathrm{p}<0.001 ; * * * *: \mathrm{p}<0.0001$.

Supplementary Figure 2: Beta diversity analysis according to age. Principal coordinate analysis of Bray-Curtis distance with each sample colored according to the studied group and stratified by age ( $<$ or $>3$ years). PC1, PC2 and PC3 represent the top three principal coordinates that captured most of the diversity. The fraction of diversity captured by the coordinate is given as a percentage. Groups were compared using ANOSIM method (9999 permutations). (A, B) all the studied groups plotted together. (C, D) TTC7A group compared to healthy subjects. (E, F) XIAP group compared to healthy subjects. *: $\mathrm{p}<0.05$; **: $\mathrm{p}<0.01$; ***: $\mathrm{p}<0.001 ; * * * *: \mathrm{p}<0.0001$. In brackets, number of samples.

\section{Supplementary Figure 3: Abnormal microbiota composition at Family level in PID.}

Bacterial diversity based on Chao1 index (A, B) in the fecal samples of indicated groups. Statistical significance was assessed using ANOVA with a post hoc Dunn’s test. *: $\mathrm{p}<0.05$; **: 
$\mathrm{p}<0.01 ; * * *: \mathrm{p}<0.001 ; * * * *: \mathrm{p}<0.0001$. Global composition of bacterial microbiota at the levels for the indicated groups (C, D).

Supplementary Figure 4: Alpha diversity and microbiota composition according to age and IBD intestinal inflammation. Bacterial diversity based on the Shannon index (A) and Chao1 index (B) in the fecal samples of indicated groups. Global composition of bacterial microbiota at the phylum (C, D) and family (E, F) levels for the indicated groups. 